\title{
El objeto de estudio de la higiene en textos publicados en España en el siglo XIX
}

\author{
The object of study of hygiene in the texts published in Spain in the $19^{\text {th }}$ century
}

Óscar A. Sánchez-Pérez y Alain R. Rodríguez-Orozco*

Facultad de Ciencias Médicas y Biológicas Dr. Ignacio Chávez, Universidad Michoacana de San Nicolás de Hidalgo, Morelia, Michoacán, México

\section{Resumen}

Con la actual pandemia de COVID-19 que la humanidad vive y la movilización de todos los sistemas de salud para ejercer medidas sanitarias, como los confinamientos, las cuarentenas y otras medidas para disminuir los contagios, vale la pena recordar que estas prácticas han sido posibles por las experiencias que en materia de higiene hemos acumulado en nuestra historia. Este ensayo tiene como objetivo destacar la higiene, la historicidad del concepto y su objeto de estudio como disciplina médica, en textos publicados en España en el siglo XIX, para ayudarnos a entender los aportes de esta ciencia a la práctica clínica actual. Con tales fines se obtuvo el material bibliográfico en la Biblioteca Nacional de España.

Palabras clave: Higiene. Historia de la medicina. Siglo XIX. Tratados de higiene.

\begin{abstract}
With the current COVID-19 pandemic that humanity is experiencing and the mobilization of all health systems to exercise sanitary measures such as quarantines and other measures to reduce infections, it should be remembered that these practices have been possible thanks to the experiences that in hygiene matters we have accumulated in our history. This essay aims to highlight about hygiene, the historicity of the concept, and its object of study as a medical discipline, in medical texts published in Spain during the $19^{\text {th }}$ century, to help us understand the contributions of this science to current clinical practice and for these purposes, the bibliographic material was obtained from the National Library of Spain.
\end{abstract}

Key words: Hygiene. History of medicine. $19^{\text {th }}$ century. Hygiene treaties.

\section{Introducción}

La actual pandemia de COVID-19 que la humanidad vive ha promovido la movilización de todos los sistemas de salud para ejercer medidas sanitarias, como los confinamientos, las cuarentenas y otras medidas, para disminuir los contagios. Vale la pena recordar que estas prácticas han sido posibles por las experiencias

\section{Correspondencia:}

*Alain R. Rodríguez-Orozco

Rafael Carrillo esquina Salvador González Herrejón s/n

Bosque Cuauhtémoc, Col. Centro

C.P. 58020, Morelia, Michoacán, México

E-mail: alain.rodriguez@umich.mx

Fecha de recepción: 09-10-2020

Fecha de aceptación: 11-01-2021

DOI: $10.24875 / C I R U .20001110$
Cir Cir. 2021;89(6):850-855

Contents available at PubMed www.cirugiaycirujanos.com 0009-7411/@ 2021 Academia Mexicana de Cirugía. Publicado por Permanyer. Este es un artículo open access bajo la licencia CC BY-NC-ND (http://creativecommons.org/licenses/by-nc-nd/4.0/). 
los fundamentos actuales de la higiene como práctica y como disciplina médica.

Se ha sugerido que las primeras experiencias con la higiene, desde la dimensión preventiva, pueden atribuirse a cuando los primeros hombres seleccionaron qué plantas y animales consumir sin que esto causara repercusiones negativas en sus cuerpos'. La palabra «higiene» tiene sus orígenes en Hygíeia (Higía), nombre que se daba a la diosa de la curación, la limpieza y la sanidad, hija de Asclepio, el dios de la medicina, y de Epione, diosa de la curación. Higía se relacionaba en específico con la prevención de enfermedades. Retomado el término por la medicina hipocrática, la higiene devino en un conjunto de normas necesarias para mantener la salud y prevenir las enfermedades ${ }^{2}$. En las civilizaciones antiguas la higiene tuvo, en sus inicios, una connotación privada, y sus prácticas se relacionaban, por ejemplo, con la alimentación, el ocio y las élites. En la Roma Antigua se practicó el syrmaismo y es importante mencionar que los emperadores romanos Tito, Trajano, Marco Aurelio y Diocleciano construyeron verdaderos complejos termales entre los siglos i y iii de nuestra era. Algunas de las obras que integran el Corpus Hippocraticum estuvieron entre los principales referentes de la higiene privada en la Antigüedad.

Los documentos especializados de higiene que más influencia tuvieron en el siglo XIX en Hispanoamérica fueron elaborados en Europa y comprendieron cartillas, compendios, manuales y tratados, basados en las teorías sobre el contagio y la propagación de enfermedades. Es posible encontrar en el mismo periodo más de una teoría de la enfermedad, porque una de las principales características del discurso médico, en particular en la segunda mitad del siglo XIX, fue el cambio de paradigma en los fundamentos científicos a partir de los trabajos realizados por Pasteur y Koch, que demostraron la existencia de seres microscópicos que eran capaces de transmitir enfermedades.

Algunos de los estudios y textos sobre la higiene más destacados del siglo XIX fueron escritos en Inglaterra, Alemania y Francia. Los textos sobre higiene escritos por expertos franceses fueron de los de mayor recepción y análisis en las escuelas de medicina y los grupos médicos del último tercio del siglo XIX en México; algunos llegaron traducidos y publicados en España. También los médicos mexicanos de la época consultaron varios textos de higienistas españoles. Debemos señalar dos cuestiones: primero, entre los médicos mexicanos del siglo XIX existía admiración por el modelo francés de salud pública, y la consulta de los textos de higiene franceses fue favorecida sobre otros textos; y segundo, en el siglo xviii en España se comenzó a implementar un nuevo modelo de medidas preventivas, que sería aplicado también para la Nueva España y que continuó en funcionamiento después de la independencia de México, tal fue el caso de las juntas sanitarias. Los textos sobre higiene provenientes de Europa permitieron a las autoridades sanitarias mexicanas del siglo XIX la emanación de medidas y la difusión de normas con el objetivo de mejorar la higiene privada y pública, como se había hecho en las sociedades modernas europeas.

Han sido temas tratados por los libros de higiene la conservación de la salud, la alimentación, la prevención de enfermedades, las formas de contagio, consejos en caso de epidemias, experiencias con el saneamiento de comunidades (como las cuarentenas y los aislamientos), e incluso el uso de remedios en comunidades. Entre un tratado y otro suele haber gran variedad en cuanto a estructura, contenido y teorías con las que se identificaban los autores para explicar, por ejemplo, los contagios o las medidas de control sanitario.

En este estudio hemos priorizado los tratados de higiene por ser los textos más completos sobre esta materia en el siglo decimonónico. El objetivo es destacar, en la higiene, la historicidad del concepto y su objeto de análisis como disciplina médica en los textos publicados en España en el siglo XIX, para ayudarnos a entender los aportes de esta ciencia a la práctica clínica actual. Algunos de los textos aquí referidos sirvieron como referentes en escuelas mexicanas de medicina, en las que el estudio y la enseñanza de la higiene como una cátedra independiente cobraría fuerza en el último tercio del siglo $\mathrm{XIX}^{3}$.

\section{La higiene en los tratados sobre la materia publicados por imprentas españolas en el siglo XIX}

Carlos Londe ${ }^{4}$, destacado miembro de la Sociedad de Medicina Práctica de París y de la Sociedad de Medicina de Londres, entre muchas otras, nos dice en el primer tomo de sus Nuevos Elementos de Higiene:

"La higiene suele definirse como el arte de conservar la salud, aunque su imperio es mucho más vasto. En efecto, es una ciencia que no se limita á prevenir los desarreglos de nuestros órganos, sino 
que además tiene por objeto su perfeccionamiento, $\mathrm{y}$ ofrece los medios mas seguros para remediar sus afecciones. Por la Higiene, pues, conserva el hombre su salud, perfecciona sus facultades, y aprende á usar y gozar de cuanto le rodea, y á evitar los peligros inseparables del abuso.»

En el Compendio de Higiene pública y privada, ó tratado elemental de los conocimientos relativos á la conservación de la Salud, y a la perfección física y moral de los hombres, su autor, M.L. Deslandes ${ }^{5}$, Doctor en Medicina de la Facultad de París y miembro del Ateneo de la Medicina, apunta:

"La higiene comprende el estudio de todas las influencias a las que está sujeto el hombre, ella nos enseña a conocer las que debemos huir y las que debemos buscar; cómo se destruyen unas y cómo se hacen favorables otras; y cómo podemos sustraernos de la acción de aquellas y aprovecharnos de los ventajosos efectos de estas. Así la higiene es una ciencia enteramente práctica, que todos los hombres necesitan conocer, y es la más importante, pues que á cada paso se ven precisados á hacer aplicaciones de ella por el primer interés de todo ser sensible, la conservación de la salud y de la vida.»

Hasta aquí encontramos dos definiciones prácticas de la higiene, que se concibe como una ciencia y por tanto tiene su objeto de estudio definido, y por otro lado se asume que permite el perfeccionamiento y nos enseña a conocer lo que debemos buscar y de lo que debemos huir. Así pues, se entiende en un sentido dinámico: sus conocimientos continuamente se actualizan con la práctica, y el perfeccionamiento de estos permitirá reconocer lo que del medio resulta favorable o desfavorable para alcanzar la salud. Por tanto, su marco referencial rebasa al del arte de conservar la salud.

La siguiente definición de higiene corresponde a la obra de Cipriano de Uribarri ${ }^{6}$, Tratado de Higiene: «la higiene estudia los medios para que se conserve el estado de salud, que es un estado de perfecto equilibrio, y continúe la vida con los atributos de bienestar en todos sus actos». En esta definición se concibe la higiene como una herramienta para llevar una vida saludable, en un estado de equilibrio que haga caso a los factores y medios que proveen una buena salud y que eran reconocidos y aceptados en esa época.

Rafael García Álvarez ${ }^{7}$, quien fuera catedrático de Historia Natural y de Fisiología e Higiene en el Instituto de Granada, en su obra Nociones de Higiene Popular nos dice acerca de la higiene lo siguiente: "es el arte de conservar la salud. Corresponde á la Medicina profiláctica, que trata de precaver las enfermedades, como la medicina terapéutica estudia el modo de curarlas, los principios en que se apoya son suministrados por las ciencias matemáticas, físicas, químicas y naturales, y particularmente por la fisiología y la medicina». En esta definición, el autor hace referencia a las ciencias particulares de las que se ha nutrido el estudio de la higiene tanto para colectar e interpretar evidencias como para proponer decisiones en materia de conservación de la salud, y hace hincapié en el sentido profiláctico de la higiene; no considera el saneamiento ambiental y la higiene privada como prácticas terapéuticas, pues para eso considera a la medicina terapéutica.

Prolegómenos de Higiene es un texto del Dr. Rafael Rodríguez Méndez ${ }^{8}$, catedrático auxiliar de la Facultad de Medicina de Granada, vocal de Sanidad y socio corresponsal de la Academia de Medicina y Cirugía de Granada y de número en Barcelona, y Catedrático de Higiene Privada y Pública de la Facultad de Barcelona. Este libro está dedicado a los estudiantes de Medicina, y por tal razón explica el autor que ha querido hacer una revisión acerca de las diferentes definiciones que han dado los más importantes higienistas de la centuria. El Dr. Rodríguez dice acerca de la higiene:

"La Higiene abarca multitud de materias, la higiene en su sentido más nato, comprende el universo entero, en tanto que diversas partes de este son capaces, directa ó indirectamente, de obrar sobre los seres vivos. Cuanto influya en los organismos, otro tanto es objeto de su estudio; de aquí resulta la multiplicidad de materias que comprende y lo fecundas y abundantes que han de ser las fuentes de sus conocimientos. El objeto final de la Higiene es la perfección orgánica, y como esto no es accesible, aspira á que cada ser se desarrolle en cuanto sea dable, de la manera más completa y acabada. Sometidos todos los organismos á la acción de inmensos modificadores, manantiales a la ves de vida y salud, muerte y enfermedad, fluctúan sin reposo, resultando de ellos impresiones, más ó ménos grandes, que engendran los tipos distintos que acepta la fisiología y estudia la patología, y de que se ocupan la física y la química cuando cesaron los fenómenos vitales. Conocer estas influencias es su trabajo especulativo (ciencia), dictar preceptos que las regulen es su aplicación (arte).»

Es esta una definición holística, con la que se aspira a una ciencia que permita la explotación de todas las capacidades del ser humano y considera la influencia que sobre el ser tienen los factores modificadores de la fisiología humana y que condicionan la 
vida y la muerte. Finalmente, señala que la higiene como disciplina teórico-práctica permite conocer las influencias de los factores que causan enfermedad, lo cual pertenece a la ciencia, pero también establecer preceptos para normar las sanas prácticas humanas, lo cual corresponde al arte.

Sobre el objeto de estudio de la higiene, el Dr. Juan Giné y Partagás ${ }^{9}$, catedrático de Clínica Quirúrgica de la Facultad de Medicina de la Universidad de BarceIona, socio de la Academia de Medicina y Cirugía de Barcelona, socio corresponsal de las academias de Medicina y Cirugía de Valencia, Sevilla, Galicia y Asturias, de la Médico-Quirúrgica Matritense, de la Sociedad Psiquiátrica de París y de la Sociedad Francesa de Higiene, en su Curso elemental de Higiene Privada y Pública en 1880 comenta:

«La higiene, partiendo de conocimientos biológicos, estudia las relaciones que para la conservación de la salud deben guardar mutuamente la organización y los medios vitales, y haciendo aplicación de estos estudios, establece reglas que tienen por objeto evitar las enfermedades, prolongar la vida y perfeccionar el ejercicio de las funciones. Pudiera, pues, decirse que la Higiene es la Fisiología aplicada á la prolongación de la vida y á la conservación de la salud: la Higiene es á la Fisiología lo que el corolario al teorema. De los teoremas fisiológicos nacen los corolarios higiénicos.»

Se registra aquí una definición del objeto de estudio de la higiene que profundiza en el aspecto relacional de esta como ciencia, cuando se dice que estudia las relaciones necesarias para conservar una vida sana y permite la generación de reglas para el automejoramiento y la preservación de la vida. Se usa el término médico «fisiopatología» para apuntar que la higiene estudia también los mecanismos que permiten la conservación de la salud, y se establece una comparación con ciencias particulares, la matemática o la lógica, para mencionar que de los teoremas fisiológicos (proposiciones lógicas susceptibles de demostración sobre el buen funcionamiento del cuerpo) nacen las conclusiones higiénicas, las cuales pudieran interpretarse como los conceptos y las normas de la higiene que demuestran la consistencia de los teoremas «fisiológicos».

De nueva cuenta se señala que en la higiene coinciden los criterios de ciencia y arte, tal como se había destacado en otras disciplinas relacionadas con la salud, pero destaca la inclusión de aspectos de la salud mental. Acerca de ello nos comenta el mismo autor ${ }^{9}$ :
«La higiene es, á la vez, ciencia y arte. Como ciencia tiene por término de sus investigaciones, por una parte el organismo, y por otra los modificadores del mismo, ora sean cósmicos, ora sean internos, ora sean del órden psíquico, y por resultado final la averiguación de las relaciones existentes entre el organismo y los medios, ó sean las leyes de las reacciones orgánicas. Como arte, da las reglas para hacer un uso conveniente y ordenado de estas mismas reacciones.»

En su tratado Higiene Doméstica, el doctor Lesmes Sánchez de Castro $^{10}$ comenta de manera concisa que «la higiene es la ciencia que tiene por objeto el cuidado de la conservación de la salud y de la vida». Una definición sencilla y operativa, accesible para cualquier público, pero para la época limitada respecto al alcance de la higiene como ciencia, quizás por estar escrita la obra para un público más general que los tratados de higiene que se publicaron en el mismo periodo. En el año 1885, el Dr. Francisco Javier Santero ${ }^{11}$, catedrático de la asignatura de Higiene Privada y Pública en la Facultad de Medicina de Madrid y Académico de la Real Academia de Medicina de Madrid, presentó su obra Elementos de Higiene privada y pública, en la cual dice que la higiene:

«es la ciencia que estudia las necesidades del hombre, y los medios de satisfacerlas conservando la salud. Teniendo por objeto la conservación de la salud y la reglamentación de las necesidades, y siendo la salud el primer bien y la primera necesidad del hombre, individual o colectivamente considerado, sólo por este hecho se comprende la importancia de su estudio y la necesidad de su vulgarización. La higiene es, pues, el primero y más indispensable de los conocimientos humanos. Por tanto, la higiene se considera como disciplina de primer orden, por tanto necesaria para alcanzar el resto de las metas, era considerada básica para solventar las necesidades del hombre. Sin higiene y sin salud resulta muy difícil cumplir con las otras metas del hombre, por tanto su conocimiento y puesta en práctica era indispensable para llevar una vida sana y productiva.»

Un año después, Francisco del Valle y Atiles $^{12}$, en su Cartilla de Higiene, hace una definición operativa y sintética que va más hacia el lado normativo y con el objetivo de la perfección humana al interpretar la higiene como «un conjunto de reglas, derivadas de las ciencias y utilizadas por el hombre para perfeccionarse y asegurar su vitalidad y la de la especie. Despréndase de esta definición que la Higiene en su 
elevado objeto no sólo abarca al individuo, sino que también al conjunto (...).»

En el año 1894 aparece publicada en dos tomos una obra de Francisco Laborde Winthuyssen ${ }^{13}$ : Lecciones de Higiene pública y privada. Este autor menciona una definición clásica y otra moderna de la higiene. A la primera corresponde «el arte de conservar la salud». Pero debe notarse otro alcance más moderno de las prácticas de la higiene, según Laborde, y se apoya en el Dr. Alcina, otro higienista del que toma la idea de que «no es posible definirla con exactitud", pero lo que está claro es que su estudio tiene por objeto «mantener las harmonías fisiológicas en su más perfecto estado".

En La higiene y la salud, de Pedro Gasalla ${ }^{14}$, se nos brindan interesantes observaciones acerca de la higiene y su importancia, y se interpreta esta como «la ciencia encargada de demostrar en donde se hallan las causas de las enfermedades, su manera de propagarse y como pueden adquirirse, para después de esto sabido, tomar las medidas necesarias para prevenirlas». Para Gasalla, prevenir las enfermedades debe ser la estrategia primaria de las prácticas de la higiene: «Esto solo puede alcanzarse llevando al ánimo de todos los más plenos convencimientos de las verdades higiénicas».

Demetrio Fidel Rubio ${ }^{15}$, en su obra Nociones elementales de Higiene, describe la higiene como:

«La ciencia y arte que estudia las causas modificadoras de la salud dando reglas ó preceptos para conservarla y perfeccionarla. Como ciencia, estudia los agentes modificadores, tanto internos como externos al organismo, las alteraciones a que dan lugar en éste y en sus mutuas relaciones. Como arte, da reglas y dirige el ejercicio de los órganos y aparatos, tendiendo al mayor perfeccionamiento de sus funciones.»

Según esta definición, a la ciencia de la higiene pertenece el estudio de factores que condicionan la pérdida de la salud y su relación con el organismo, y al arte de la higiene, las reglas para el perfeccionamiento de las funciones del organismo.

Otra definición de higiene nos la ofrece Juan M. Díaz Villar ${ }^{16}$ en su Tratado elemental de higiene comparada del hombre y los animales domésticos. En ella se precisa que:

«La Higiene, rama esencial de la Medicina, es la colección de reglas que tiene por fin inmediato o próximo la preservación de las enfermedades, y por fin mediato o remoto, la conservación de la salud. La Higiene, por consiguiente, tiende a aumentar la sana longevidad del hombre y de los animales domésticos.»
En esta definición se reconoce a la higiene como disciplina médica que tiene como fin la conservación de la salud, y no como un simple conjunto de normas o el estudio de mecanismos aislados de interacción del organismo con factores que propician la enfermedad, y se asume su carácter profiláctico. Esta definición implica, según el autor, el rumbo que debe seguirse en las aplicaciones de las reglas higiénicas, con el fin primario de evitar enfermedades, y como fin secundario o consecuencia de la ausencia del mal, la prolongación de la vida en condiciones cómodas y agradables.

En una obra premiada en la Exposición del IX Congreso de Higiene celebrado en Madrid en el año de 1898, Nociones de Higiene y Fisiología, su autora, Luciana Casilda Monreal ${ }^{17}$, dice que la higiene «es la parte de la Medicina que nos da reglas para conservar la salud, la cual consiste en el funcionamiento ordenado de todos los órganos del cuerpo». Y aclara que la higiene, para establecer sus preceptos, ha de tener en cuenta el sexo, los temperamentos, las idiosincrasias, la herencia, los hábitos, la constitución y la edad del individuo. Llama la atención en este postulado la identificación de factores demográficos, familiares y culturales que apuntan a la necesidad de analizar las prácticas de la higiene en un contexto sociocultural, algo realmente vanguardista para la época.

El reconocimiento de factores modificadores de la salud es un hecho ancestral, y algunos de estos factores ya habían sido interpretados por Galeno, quien reconocía la influencia de «lo que está dentro o fuera de nosotros y que suelen llamarse naturales, no naturales y extranaturales». A los primeros pertenecen los sólidos y fluidos que conforman el cuerpo humano; a los segundos, las cosas que influyen sobre el hombre, como el aire, la comida y la bebida, el movimiento y la quietud, los afectos del ánimo o la apatía, el sueño y la vigilia, y las secreciones, expelidas o detenidas; y a los terceros, las enfermedades ${ }^{6}$.

La obra Précis d'hygiene privée et sociale, de Alexandre Lacassagne ${ }^{18}$, tiene una connotación adicional, ya que fue el libro de texto utilizado para impartir la cátedra de higiene que formaba parte de la carrera de medicina en la ciudad de Morelia en la década de 1880. Lacassagne dice:

«La higiene es ordinariamente definida como el arte de conservar la salud, si esto es cierto como lo dice la sabiduría antigua, que la salud es la primera propiedad del hombre, la higiene debe ser la primera de las artes; es una de las ramas más importantes de la medicina.»

En 1868 se separó la cátedra de Higiene de la de Fisiología en la Escuela Nacional de Medicina en la 
Ciudad de México, y recibió el nombre de Higiene y Meteorología Médica, siendo sus primeros profesores Ladislao de la Pascua, José G. Lobato y Luis E. Ruiz. Este último tomó posesión de dicha cátedra en 1889 y consideró que la higiene era el arte científico de conservar la salud y vigorizar el organismo, y como otros higienistas que le antecedieron insistió en que la higiene es «la primera de las artes, puesto que la salud es el primero de los bienes ${ }^{19}$. Llama la atención en su curso de higiene la contextualización de los preceptos de la higiene, individual y social, a las condiciones naturales y sociales de la ciudad de México, donde eran distinguibles estudios de los suelos, los gases, el agua, las adicciones, las epidemias y las estadísticas de población en congruencia con la etnografía y la antropología vigentes en el siglo XIX ${ }^{19}$. Para esta noble tarea como educador médico debió integrar y aplicar los conocimientos de higiene vigentes hasta el siglo XIX, provenientes de los tratados de higiene europeos, a las condiciones de fines del siglo XIX en la Ciudad de México.

\section{Conclusiones}

Las definiciones de higiene que muestran los tratados publicados en España en el siglo XIX nos han dejado claro que, durante la mayor parte de ese siglo, los higienistas se mantuvieron preocupados por algunas cuestiones como la conciliación entre arte y ciencia, su naturaleza profiláctica, su carácter de ciencia primaria de los cuidados para posibilitar con la salud el cumplimiento de las otras metas del ser humano, su papel dinámico porque se actualiza continuamente con sus prácticas, y por tanto se nutre con la experiencia, por ejemplo «de las pandemias y de la eficacia o ineficacia de las medidas de saneamiento ambiental en las comunidades humanas", y la investigación de factores internos y externos al humano que condicionan la enfermedad o ayudan a prevenir de algún mal, a los cuales se llamó «influencias, agentes o modificadores». Es necesario que reconozcamos el papel trascendente que los estudios de la higiene en el siglo XIX han tenido sobre la evolución histórica de la medicina. Antes de la diversificación de la práctica clínica en múltiples especialidades, la higiene fue el tronco común que permitió recolectar las experiencias que ayudaron al hombre a perfeccionarse, aumentar su calidad de vida e incluso sobrevivir a pestes y epidemias. Sí, estamos de acuerdo con el criterio de algunos de los higienistas del siglo XIX, que la entendieron como una disciplina médica primaria, porque su práctica era necesaria para obtener una salud que permitiera cumplir con las otras metas de la vida del hombre, y también porque con su práctica fue posible nuestra supervivencia como especie y porque, como disciplina primero de los cuidados y luego médica, fue probablemente una de las primeras en reconocer los factores «internos y externos» que modifican el binomio salud-enfermedad y en usarlos con fines profilácticos.

\section{Conflicto de intereses}

Los autores declaran no tener conflicto de intereses alguno.

\section{Responsabilidades éticas}

Protección de personas y animales. Los autores declaran que para esta investigación no se han realizado experimentos en seres humanos ni en animales.

Confidencialidad de los datos. Los autores declaran que en este artículo no aparecen datos de pacientes.

Derecho a la privacidad y consentimiento informado. Los autores declaran que en este artículo no aparecen datos de pacientes.

\section{Bibliografía}

1. Lain P. Historia Universal de la Medicina. Tomo I. Barcelona: Salvat; 1974. p. 29.

2. Quevedo E. Cuando la higiene se volvió pública. Rev Fac Med. 2004;52:83-5.

3. Martínez-Cortés F. La medicina científica y el siglo XIX mexicano. La ciencia para todos. México: Fondo de Cultura Económica; 1997. p. 138.

4. Londe C. Nuevos elementos de higiene, Tomo I. Madrid; 1829. p. 30.

5. Deslandes ML.Compendio de higiene pública y privada. Tomo I. Gerona; 1829. p. $19-20$.

6. De Uribarri C. Tratado de higiene. Barcelona: Imprenta y Librería de Tomás de Gorchs; 1852. p. 9.

7. García-Álvarez R. Nociones de higiene popular. Granada: Imprenta Ventura; 1874. p. 13

8. Rodríguez-Méndez R. Prolegómenos de higiene. Barcelona: Establecimiento Tipográfico de Jaime Jepús; 1874. p. 4.

9. Giné y Partagás J. Curso elemental de higiene privada y pública. Barcelona: Juan y Antonio Bastinos; 1880. p. 22.

10. Sánchez de Castro L. Higiene doméstica. León; 1882. p. 21.

11. Santero J.F. Elementos de higiene pública y privada. Tomo I. Madrid: EI Cosmos; 1885. p. 12.

12. Del Valle y Atiles F. Cartilla de higiene. Puerto Rico: Imp. de José González Font; 1886. p. 12.

13. Laborde y Winthuyssen F. Lecciones de higiene privada y pública. Tomo I. Sevilla: Imprenta de Díaz y Carballo; 1894. p. 23-4.

14. Gasalla P. La higiene y la salud. Lugo; 1894. p. 11-2.

15. Rubio y Alberto DF. Nociones elementales de higiene. Madrid; 1895. p. 9.

16. Díaz Villar y Martínez JM. Tratado elemental de higiene comparada del hombre y los animales domésticos. Madrid: Imprenta de Enrique Teodoro; 1896. p. 41.

17. Monreal LC. Nociones de higiene y fisiología. 3. ${ }^{-}$ed. Madrid: Avrial; 1903. p. 5-6.

18. Lacassagne A. Précis d'hygiène privée et sociale. Paris: Masson; 1876. p. 12.

19. Martínez-Cortés F. La medicina científica y siglo XIX mexicano. En: La higiene. 2. ${ }^{a}$ ed. Ciudad de México: Fondo de Cultura Económica; 1997. Capítulo VII. 